PROCEEDINGS OF THE

AMERICAN MATHEMATICAL SOCIETY

Volume 131, Number 9, Pages 2753-2759

S 0002-9939(03)07011-4

Article electronically published on April 7, 2003

\title{
$p$-HYPONORMAL OPERATORS ARE SUBSCALAR
}

\author{
LIN CHEN, RUAN YINGBIN, AND YAN ZIKUN
}

(Communicated by Joseph A. Ball)

\begin{abstract}
We prove that if $R, S \in B(\mathbf{X}), R, S$ are injective, then $R S$ is subscalar if and only if $S R$ is subscalar. As corollaries, it is shown that $p$ hyponormal operators $(0<p \leq 1)$ and log-hyponormal operators are subscalar; also w-hyponormal operators $T$ with $\operatorname{Ker} T \subset \operatorname{Ker} T^{*}$ and their generalized Aluthge transformations $T(r, 1-r)(0<r<1)$ are subscalar.
\end{abstract}

\section{INTRODUCTION AND NOTATIONS}

Let $\mathbf{X}$ denote a Banach space. $T \in B(\mathbf{X})$ is said to be generalized scalar ( 1 ) if there exists a continuous algebra homomorphism

$$
\Phi: \quad \varepsilon(\mathbb{C}) \longrightarrow B(\mathbf{X})
$$

with $\Phi(1)=I$ and $\Phi(z)=T$. Here $\varepsilon(\mathbb{C})$ denotes the algebra of all infinitely differentiable functions on $\mathbb{C}$ with the topology defined by the uniform convergence of such functions and their derivatives ([2]). An operator similar to the restriction of a generalized scalar operator to one of its closed invariant subspaces is called subscalar. Subscalar operators are subdecomposable ([1]).

Let $\mathbf{H}$ denote an infinite-dimensional separable Hilbert space, $T \in B(\mathbf{H})$. $T$ is said to be $p$-hyponormal $(p>0)$ if $\left(T^{*} T\right)^{p} \geq\left(T T^{*}\right)^{p}$, i.e., $|T|^{2 p} \geq\left|T^{*}\right|^{2 p}$. A $p$-hyponormal operator is called hyponormal if $p=1$ and semi-hyponormal if $p=\frac{1}{2}$ ([3], 4]). The Löwner-Heinz inequality implies that if $T$ is $q$-hyponormal, then it is $p$-hyponormal for any $0<p \leq q$. An invertible operator $T$ is said to be $\log$-hyponormal ([5]) if $\log \left(T^{*} T\right) \geq \log \left(T T^{*}\right)$.

For $T \in B(\mathbf{H})$, let $T=U|T|$ be the polar decomposition of $T$, and for $s, t \geq 0$, $T(s, t)=|T|^{s} U|T|^{t}$ is called the generalized Aluthge transformation of $T$. $T\left(\frac{1}{2}, \frac{1}{2}\right)=$ $|T|^{\frac{1}{2}} U|T|^{\frac{1}{2}}$ is denoted by $\hat{T}$. It is known that if $T$ is $p$-hyponormal $(p>0)$, then $\hat{T}$ is semihyponormal and $(\hat{\hat{T}})=\hat{T}\left(\frac{1}{2}, \frac{1}{2}\right)$ (denoted by $\tilde{T}$ ) is hyponormal. If $T$ is log-hyponormal, then $\hat{T}$ is semihyponormal. $T$ is said to be w-hyponormal ([6]) if $|\hat{T}| \geq|T| \geq\left|\hat{T}^{*}\right|$, so $\hat{T}$ is semihyponormal if $T$ is w-hyponormal. It is shown in [6] that $p$-hyponormal $(p>0)$ and log-hyponormal operators are w-hyponormal.

Received by the editors February 12, 2002.

2000 Mathematics Subject Classification. Primary 47B99, 47A10.

Key words and phrases. Subscalar, p-hyponormal, log-hyponormal, w-hyponormal, Aluthge transformations.

This research was supported by the National Natural Science Foundation of China. 
M. Putinar showed ([7]) that hyponormal operators are subscalar; Y. Chu showed (8]) that semihyponormal operators are also subscalar. Are all $p$-hyponormal $(p>$ 0 ) operators subscalar? Some authors have discussed this problem and obtained certain partial answers. We are going to derive a result: if $R, S \in B(\mathbf{X}), R, S$ injective, then $R S$ is subscalar if and only $S R$ is subscalar. Appling this result to generalized Aluthge transformation of operators, we get an affirmative and stronger answer to the above question: all p-hyponormal operators and log-hyponormal operators are subscalar; w-hyponormal operators $T$ with $\operatorname{Ker} T \subset \operatorname{Ker} T^{*}$ and their generalized Aluthge transformation $T(r, 1-r) \quad(0<r<1)$ are also subscalar.

\section{MAin Results}

Let $\mathbf{X}$ denote a Banach space. $R, S \in B(\mathbf{X})$, operators $R S$ and $S R$ have many common properties, including spectral properties, subdecomposability, nontriviality of invariant and hyperinvariant subspaces, etc. (see [9, [10]). It is proved in 9 that if $R, S$ are injective, then $R S$ is subdecomposable if and only if $S R$ is subdecomposable, whence one can derive that $p$-hyponormal operators $(p>0)$ are subdecomposable (see also [11]). We are now going to present a similar result for subscalarity.

Theorem A. If $R, S \in B(\mathbf{X})$, where $R$ and $S$ are injective, then $R S$ is subscalar if and only if $S R$ is subscalar.

Some lemmas are to be considered first. $\varepsilon(U, \mathbf{X})$ denotes the Fréchet space of all $\mathbf{X}$-valued $C^{\infty}$-functions, i.e., infinitely continuously differentiable functions on $U$ ([1]). $T$ is said to have property $(\beta)_{\varepsilon}$ (denoted by $\left.T \in(\beta)_{\varepsilon}\right)$ if for each open set $U$ in $\mathbb{C}$ the operator

$$
T_{z}: \quad \varepsilon(U, \mathbf{X}) \longrightarrow \varepsilon(U, \mathbf{X}), \quad f \longmapsto(T-z) f
$$

is a topological monomorphism, i.e., $T_{z} f_{n} \rightarrow 0$ implies $f_{n} \rightarrow 0$ for $f_{n} \in \varepsilon(U, \mathbf{X})$.

Lemma 1. For $T \in B(\mathbf{X})$, the following statements are equivalent:

(1) $T$ is subscalar;

(2) $T \in(\beta)_{\varepsilon}$, i.e., for each open subset $U$ of $\mathbb{C}, f_{n} \in \varepsilon(U, \mathbf{X}),(T-z) f_{n} \longrightarrow$ $0 \quad(n \rightarrow \infty)$ implies $f_{n} \longrightarrow 0 \quad(n \rightarrow \infty)$;

(3) $T_{z}=T-z$ acts one-to-one and with closed range on $\varepsilon(U, \mathbf{X})$ for each open subset $U$ of $\mathbb{C}$.

Proof. $(1) \Longleftrightarrow(2)$. See corollary 4.6 of $[1]$.

$(2) \Longrightarrow(3)$. Suppose $f \in \varepsilon(U, \mathbf{X}), T_{z} f=0$. Take $f_{n}=f \quad(n=1,2, \cdots)$; then $T_{z} f_{n} \longrightarrow 0(n \rightarrow \infty), f=\lim _{n \rightarrow \infty} f_{n}=0$. $T_{z}$ is one-to-one on $\varepsilon(U, \mathbf{X})$.

Suppose that $f_{n}, g \in \varepsilon(U, \mathbf{X}), \lim _{n \rightarrow \infty} T_{z} f_{n}=g$. Then $T_{z}\left(f_{n}-f_{m}\right) \longrightarrow$ $0(n, m \rightarrow \infty)$. This implies that $f_{n}-f_{m} \longrightarrow 0(n, m \rightarrow \infty)$ and $f_{n} \longrightarrow f \in$ $\varepsilon(U, \mathbf{X})(n \rightarrow \infty)$ since the Fréchet space $\varepsilon(U, \mathbf{X})$ is complete. It follows that $g=\lim _{n \rightarrow \infty} T_{z} f_{n}=T_{z} f$. Therefore the range of $T_{z}$ is closed.

$(3) \Longrightarrow(2)$. Suppose that $f_{n} \in \varepsilon(U, \mathbf{X}), n=1,2, \cdots, T_{z} f_{n} \longrightarrow 0(n \rightarrow \infty)$. Let $E=\left\{T_{z} f: f \in \varepsilon(U, \mathbf{X})\right\} ; \mathbf{E}$ is a closed subspace of $\varepsilon(U, \mathbf{X})$ and is therefore a Fréchet space, too. $S=T_{z}: \varepsilon(U, \mathbf{X}) \longrightarrow E$ is one-to-one and subjective. In view that $S$ is a continuous linear mapping from $\varepsilon(U, \mathbf{X}) \longrightarrow \mathbf{E}$, the open mapping theorem implies that $S^{-1}$ is continuous and hence that $f_{n}=S^{-1} T_{z} f_{n} \longrightarrow 0(n \rightarrow \infty)$. 
Lemma 2. Let $U \subset \mathbb{C}, U$ be open, $0 \in U ; g(z) \in \varepsilon(U, \mathbf{X}), \varphi(z) \in \varepsilon(U \backslash\{0\}, \mathbf{X})$, $Q \in B(\mathbf{X})$ being injective, $g(z)=(Q-z) \varphi(z), z \in U \backslash\{0\} .\left\{z_{n}\right\}$ and $\left\{t_{n}\right\}$ are two sequences in $\mathbb{C}$ converging to 0 . Let $(1) \Phi(z)=\varphi(z)$ or $(2) \Phi(z)=\frac{\partial^{k} \varphi(z)}{\partial u_{1} \cdots \partial u_{k}}\left(u_{i}=x\right.$ or $y, i=1,2, \cdots, k), \varphi$ and its derivatives of order $n<k$ are continuous at $z=0$. If $\lim _{n \rightarrow \infty} \Phi\left(z_{n}\right)$ and $\lim _{n \rightarrow \infty} \Phi\left(t_{n}\right)$ both exist, then $\lim _{n \rightarrow \infty} \Phi\left(z_{n}\right)=\lim _{n \rightarrow \infty} \Phi\left(t_{n}\right)$.

Proof. (1) $\Phi(z)=\varphi(z)$. By hypothesis,

$$
g\left(z_{n}\right)=\left(Q-z_{n}\right) \varphi\left(z_{n}\right), g(0)=\lim _{n \rightarrow \infty} g\left(z_{n}\right)=Q \lim _{n \rightarrow \infty} \varphi\left(z_{n}\right) .
$$

Similarly $g(0)=Q \lim _{n \rightarrow \infty} \varphi\left(t_{n}\right)$. The injectivity of $Q$ then implies that

$$
\lim _{n \rightarrow \infty} \Phi\left(z_{n}\right)=\lim _{n \rightarrow \infty} \Phi\left(t_{n}\right) .
$$

(2) $\Phi(z)=\frac{\partial^{k} \varphi(z)}{\partial u_{1} \cdots \partial u_{k}}$ It is clear by calculation and induction that

$$
G(z)=\frac{\partial^{k} g(z)}{\partial u_{1} \cdots \partial u_{k}}=(Q-z) \Phi(z)-\sum_{i=1}^{m} a_{i} \psi_{i}(z), \quad z \in U \backslash\{0\} .
$$

Here $\psi_{i}=\varphi$ or derivatives of $\varphi$ of order $n<k, a_{i}$ are constants, $1 \leq i \leq m$. Since $g \in \varepsilon(U, \mathbf{X})$, hence

$$
\begin{aligned}
& G(0)=\lim _{n \rightarrow \infty} G\left(z_{n}\right)=Q \lim _{n \rightarrow \infty} \Phi\left(z_{n}\right)-\sum_{i=1}^{m} a_{i} \lim _{n \rightarrow \infty} \psi_{i}\left(z_{n}\right), \\
& G(0)=\lim _{n \rightarrow \infty} G\left(t_{n}\right)=Q \lim _{n \rightarrow \infty} \Phi\left(t_{n}\right)-\sum_{i=1}^{m} a_{i} \lim _{n \rightarrow \infty} \psi_{i}\left(t_{n}\right) .
\end{aligned}
$$

The continuity of $\psi_{i}$ at $z=0$ implies that $\lim _{n \rightarrow \infty} \psi_{i}\left(z_{n}\right)=\lim _{n \rightarrow \infty} \psi_{i}\left(t_{n}\right)$, $1 \leq i \leq m$. Consequently we have $Q \lim _{n \rightarrow \infty} \Phi\left(z_{n}\right)=Q \lim _{n \rightarrow \infty} \Phi\left(t_{n}\right)$ and $\lim _{n \rightarrow \infty} \Phi\left(z_{n}\right)=\lim _{n \rightarrow \infty} \Phi\left(t_{n}\right)$ by the injectivity of $Q$.

Lemma 3. Let $0 \in U \subset \mathbb{C}, U$ be open, $h(z), g(z) \in \varepsilon(U, \mathbf{X}), h(0)=0, Q \in B(\mathbf{X})$ be injective. If $g(z)=(Q-z) \frac{h(z)}{z}, \quad z \in U \backslash\{0\}$, then we can define

$$
\varphi(z)= \begin{cases}h(z) / z, & z \in U \backslash\{0\}, \\ y_{0}, & z=0\end{cases}
$$

$\left(y_{0} \in \mathbf{X}\right)$, such that $\varphi(z) \in \varepsilon(U, \mathbf{X})$.

Proof. It follows from the Taylor formula that

$$
h(z)=x h_{x}^{\prime}(0)+y h_{y}^{\prime}(0)+R(z), \quad z \in U .
$$

Here $R(z)=o(|z|)$, i.e., $\frac{R(z)}{|z|} \longrightarrow 0(z \rightarrow 0)$. Hence

$$
\varphi(z)=\frac{h(z)}{z}=\frac{x h_{x}^{\prime}(0)+y h_{y}^{\prime}(0)}{x+i y}+\frac{R(z)}{z}, \quad z \in U \backslash\{0\} .
$$

By (1), $\lim _{x \rightarrow 0} \varphi(x)=\lim _{x \rightarrow 0} \frac{h(x)}{x}=h_{x}^{\prime}(0)$ and $\lim _{y \rightarrow 0} \varphi(i y)=\lim _{y \rightarrow 0} \frac{h(i y)}{i y}=$ $\frac{h_{y}^{\prime}(0)}{i}$ both exist. Lemma 2 implies that $h_{x}^{\prime}(0)=\frac{h_{y}^{\prime}(0)}{i}$, whence

$$
\begin{aligned}
\varphi(z) & =\frac{h_{x}^{\prime}(0)(x+i y)}{x+i y}+\frac{R(z)}{z} \\
& =h_{x}^{\prime}(0)+\frac{R(z)}{z}, \quad z \in U \backslash\{0\} .
\end{aligned}
$$


Define $y_{0}=h_{x}^{\prime}(0)$; then we have

$$
\varphi(z)= \begin{cases}h_{x}^{\prime}(0)+\frac{R(z)}{z}, & z \in U \backslash\{0\}, \\ h_{x}^{\prime}(0), & z=0,\end{cases}
$$

$R(z) \in \varepsilon(U, \mathbf{X}), \quad R(z)=o(|z|)$ and the continuity of $\varphi(z)$ in $U$ follows at once.

Suppose that $\Phi$ is a $k$ th order derivative of $\varphi$ (including $\varphi^{(0)}=\varphi$ ) and that $\varphi$ and all derivatives of $\varphi$ of order $n \leq k$ are continuous at $z=0$. $\Phi$ satisfies the condition

$$
\Phi(z)= \begin{cases}a+\frac{G(z)}{z^{k+1}}, & z \in U \backslash\{0\}, \\ a, & z=0,\end{cases}
$$

$G(z) \in \varepsilon(U, \mathbf{X}), G(z)=o\left(|z|^{k+1}\right), a \in \mathbf{X}$ being a constant vector.

We are going to show that $\Phi_{x}^{\prime}$ and $\Phi_{y}^{\prime}$ exist and are continuous at $z=0$ and satisfy condition $\left(C_{k+1}\right)$ similar to $\left(C_{k}\right)$.

Applying the Taylor formula, we have

$$
\begin{gathered}
G(z)=G(0)+G_{1}(z)+\cdots+G_{i}(z)+\cdots+G_{k+2}(z)+R_{1}(z), \\
G_{i}(z)=\frac{1}{i !}\left(x \frac{\partial}{\partial x}+y \frac{\partial}{\partial y}\right)^{i} G(0), 1 \leq i \leq k+2, R_{1}(z)=o\left(|z|^{k+2}\right) .
\end{gathered}
$$

The hypothesis $G(z)=o\left(|z|^{k+1}\right)$ implies that

$$
G(0) \equiv G_{1}(z) \equiv \cdots \equiv G_{k+1}(z) \equiv 0
$$

and hence that

$$
\frac{\partial^{n} G}{\partial^{m} x \partial^{n-m} y}(0)=0, \quad 1 \leq n \leq k+1, \quad 0 \leq m \leq n .
$$

It follows from (2) and (3) that

$$
G(z)=\frac{G_{x}^{(k+2)}(0)}{(k+2) !} x^{k+2}+a_{1} x^{k+1} y+\cdots+a_{k+2} y^{k+2}+R_{1}(z) .
$$

Here $a_{1}, a_{2}, \cdots, a_{k+2}$ are constant vectors.

Similarly we have (noticing that $G_{x}^{\prime}(z) \in \varepsilon(U, \mathbf{X})$, too), by (3),

$$
\begin{aligned}
G_{x}^{\prime}(z) & =\frac{1}{(k+1) !}\left(x \frac{\partial}{\partial x}+y \frac{\partial}{\partial y}\right)^{k+1} G_{x}^{\prime}(0)+R_{2}(z) \\
& =\frac{G_{x}^{k+2}(0)}{(k+1) !} x^{k+1}+b_{1} x^{k} y+\cdots+b_{k+1} y^{k+1}+R_{2}(z) .
\end{aligned}
$$

Here $b_{1}, b_{2}, \cdots, b_{k+1}$ are constant vectors, $R_{2}(z)=o\left(|z|^{k+1}\right)$.

From (4) and (5), we obtain

$$
\Phi_{x}^{\prime}(0)=\lim _{x \rightarrow 0} \frac{\Phi(x)-\Phi(0)}{x}=\lim _{x \rightarrow 0} \frac{G(x)}{x^{k+2}}=\frac{G_{x}^{(k+2)}(0)}{(k+2) !}=a_{0},
$$

say, and

$$
\begin{aligned}
\Phi_{x}^{\prime}(z) & =\frac{z G^{\prime}(z)-(k+1) G(z)}{z^{k+2}} \\
& =\frac{a_{0} x^{k+2}+\alpha_{1} x^{k+1} y+\cdots+\alpha_{k+2} y^{k+2}}{(x+i y)^{k+2}}+\frac{F(z)}{z^{k+2}} \quad(z \in U \backslash\{0\}) .
\end{aligned}
$$


Here $\alpha_{1}, \alpha_{2}, \cdots, \alpha_{k+2}$ are constant vectors, $F(z)=z R_{2}(z)-(k+1) R_{1}(z)=$ $o\left(|z|^{k+2}\right)$.

Substituting $x=r \cos \theta, y=r \sin \theta$ into the first term of the right-hand side, we obtain

$$
\Phi_{x}^{\prime}(z)=f(\theta)+\frac{F(z)}{z^{k+2}}, \quad z \in U \backslash\{0\}
$$

$\lim _{r \rightarrow 0} \Phi_{x}^{\prime}(z)=\lim _{r \rightarrow 0} \Phi_{x}^{\prime}[r(\cos \theta+i \sin \theta)]=f(\theta)$ exists for every $\theta, 0 \leq \theta \leq 2 \pi$, therefore it follows from Lemma 2 that

$$
f(\theta)=f(0)=a_{0}=\Phi_{x}^{\prime}(0) .
$$

$\Phi_{x}^{\prime}(z)$ satisfies the following condition:

$\left(C_{k+1}\right) \quad \Phi_{x}^{\prime}(z)= \begin{cases}a_{0}+\frac{F(z)}{z}, & z \in U \backslash\{0\}, \\ a_{0}, & z=0,\end{cases}$

$F(z) \in \varepsilon(U, \mathbf{X}), F(z)=o\left(|z|^{k+2}\right)$, whence $\Phi_{x}^{\prime}$ is continuous at $z=0$.

Similarly we have that $\Phi_{y}^{\prime}$ exists and is continuous at $z=0$ and satisfies

$\left(C_{k+1}\right) \quad \Phi_{y}^{\prime}(z)= \begin{cases}b_{0}+\frac{E(z)}{z^{k+2}}, & z \in U \backslash\{0\} \\ b_{0}, & z=0\end{cases}$

$E(z) \in \varepsilon(U, \mathbf{X}), E(z)=o\left(|z|^{k+2}\right)$, here $b_{0}$ being a constant vector.

We have proved that $\varphi^{(0)}=\varphi$ is continuous at $z=0$ and satisfies condition $\left(C_{0}\right)$. It follows by mathematical induction that $\varphi$ has continuous derivatives of any order at $z=0$, i.e., $\varphi \in \varepsilon(U, \mathbf{X})$.

Proof of Theorem A. By Lemma 1, it is sufficient to show that if $R$ and $S$ are injective and $R S-z$ is one-to-one and has closed range, then $S R-z$ is one-to-one and has closed range, too.

Let $U$ be any open subset of $\mathbb{C}, F(z) \in \varepsilon(U, \mathbf{X}),(S R-z) F(z)=0$. It follows that

$$
(R S-z) R F(z)=R(S R-z) F(z)=0 \quad(z \in U)
$$

Since $R S-z$ is one-to-one, hence $R F(z)=0$ and the injectivity of $R$ implies $F(z)=0(z \in U)$ so that $S R-z$ is one-to-one on $\varepsilon(U, \mathbf{X})$.

Suppose that $g \in \varepsilon(U, \mathbf{X}), f_{n} \in \varepsilon(U, \mathbf{X})(n=1,2, \cdots)$ and $(S R-z) f_{n}(z) \longrightarrow$ $g(z)$ on $\varepsilon(U, \mathbf{X})$. Then $(R S-z) R f_{n}(z)=R(S R-z) f_{n}(z) \longrightarrow R g(z) \in \varepsilon(U, \mathbf{X})$. Since $R S-z$ has closed range, there exists $f(z) \in \varepsilon(U, \mathbf{X})$ such that $R g(z)=$ $(R S-z) f(z)$ and hence

$$
R S f(z)=R g(z)+z f(z)
$$

Let $h(z)=S f(z)-g(z)$. Then $h(z) \in \varepsilon(U, \mathbf{X})$ and

$$
\begin{gathered}
R h(z)=R(S f(z)-g(z))=z f(z) \\
(S R-z) h(z)=S[z f(z)]-z S f(z)+z g(z)=z g(z) \\
(S R-z) \frac{h(z)}{z}=g(z), \quad z \in U \backslash\{0\} .
\end{gathered}
$$

If $0 \notin U$, then $\frac{h(z)}{z} \in \varepsilon(U, \mathbf{X}), g(z)$ belongs to the range of $S R-z$ on $\varepsilon(U, \mathbf{X})$, that is, $S R-z$ has closed range on $\varepsilon(U, \mathbf{X})$. 
If $0 \in U$, then by $(6), R g(0)=R S f(0), g(0)=S f(0)$, hence $h(0)=S f(0)-$ $g(0)=0$. By Lemma 3 , taking $Q=S R$, we can define

$$
\varphi(z)= \begin{cases}\frac{h(z)}{z}, & z \in U \backslash\{0\} \\ y_{0}, & z=0\end{cases}
$$

such that $\varphi(z) \in \varepsilon(U, \mathbf{X})$. By (7) and the continuity of $\varphi$ at $z=0$, we have

$$
(S R-z) \varphi(z)=g(z), \quad z \in U,
$$

and hence that $S R-z$ has closed range on $\varepsilon(U, \mathbf{X})$. The conclusion that $S R$ is subscalar now follows.

Now let us turn to the applications of Theorem A. Let $\mathbf{H}$ denote a separable Hilbert space in the sequel.

Corollary 1. Let $T \in B(\mathbf{H}), \operatorname{Ker} T \subset K e r T^{*}, s \geq 0,0<r<t$. Then $T(s, t)$ is subscalar if and only if $T(s+r, t-r)$ is subscalar; especially $T=T(0,1)$ is subscalar if and only if $T(r, 1-r)(0<r<1)$ is subscalar.

Proof. Since $\operatorname{Ker} T \subset \operatorname{Ker} T^{*}, \operatorname{Ker} T$ reduces $T$ and $T=\theta \oplus T_{1}$; here $\theta=\left.T\right|_{\operatorname{Ker} T}, T_{1}=$ $\left.T\right|_{(\operatorname{Ker} T)^{\perp}}, \operatorname{Ker} T_{1}=\{0\}$. It is clear that $T(\alpha, \beta)=\theta \oplus T_{1}(\alpha, \beta)(\alpha, \beta>0)$. Suppose that $T_{1}=U_{1}\left|T_{1}\right|$ is the polar decomposition of $T_{1}, R=\left|T_{1}\right|^{r}, S=\left|T_{1}\right|^{s} U_{1}\left|T_{1}\right|^{t-r}$; then $\operatorname{Ker} R=\operatorname{Ker} S=\{0\}, R S=T_{1}(s+r, t-r), S R=T_{1}(s, t)$. By Theorem A, it concludes that $T_{1}(s, t)$ is subscalar if and only if $T_{1}(s+r, t-r)$ is subscalar and hence that $T(s, t)$ is subscalar if and only if $T(s+r, t-r)$ is subscalar.

Corollary 2. Let $T \in B(\mathbf{H})$ be a p-hyponormal $(0<p \leq 1)$ operator or a loghyponormal operator. Then $T$ is subscalar.

Proof. Suppose $T$ is a $p$-hyponormal $(0<p \leq 1)$ operator. Then we have $\operatorname{Ker} T \subset$ $\operatorname{Ker} T^{*}$ ([12], Lemma 1), $\tilde{T}=\hat{T}\left(\frac{1}{2}, \frac{1}{2}\right)$ is hyponormal and therefore subscalar ([7]). By Corollary 1, this implies that $\hat{T}=T\left(\frac{1}{2}, \frac{1}{2}\right)$ and $T=T(0,1)$ are subscalar.

Suppose $T$ is a $\log$-hyponormal operator. Then $T$ is invertible, $\operatorname{Ker} T=\operatorname{Ker} T^{*}=$ $\{0\}$. Since $\hat{T}=T\left(\frac{1}{2}, \frac{1}{2}\right)$ is $\frac{1}{2}$-hyponormal and therefore subscalar, $T$ is also subscalar by Corollary 1 .

Corollary 3. Let $T \in B(\mathbf{H})$ be w-hyponormal and $\operatorname{Ker} T \subset K e r T^{*}$. Then $T$ and their generalized Aluthge transformation $T(r, 1-r)(0<r<1)$ are subscalar.

Proof. By definition, $|\hat{T}| \geq\left|\hat{T}^{*}\right|$. This implies that $\hat{T}=T\left(\frac{1}{2}, \frac{1}{2}\right)$ is $\frac{1}{2}$-hyponormal and subscalar. Since $\operatorname{Ker} T \subset \operatorname{Ker} T^{*}$, it follows from Corollary 1 that $T$ and $T(r, 1-r)(0<r<1)$ are subscalar.

\section{REFERENCES}

1. J. Eschmeier and M. Putinar, Bishop's condition $(\beta)$ and rich extensions of linear operators, Indiana Univ. Math. J. 37 (1988), 325-348. MR 89k:47051

2. I. Colojoară and C. Foias, Theory of generalized spectral operators, New York, Gordon and Breach,, 1968. MR 52:15085

3. A. Aluthge, On p-hyponormal operators for $0<p<1$, Integr. Equat. Oper. Th. 13 (1990), 307-315. MR 91a:47025

4. D. Xia, Spectral theory of hyponormal operators, Birkhäuser Verlag, Basel, 1983. MR $\mathbf{8 7 j}: 47036$

5. Kôtarô Tanahashi, On log-hyponormal operators, Integr. Equat. Oper. Th. 34 (1999), 364-372. MR 2000b:47055 
6. A. Aluthge and D. Wang, w-Hyponormal operators II, Integr. Equat. Oper. Th. 37 (2000), 324-331. MR 2001i: 47032

7. M. Putinar, Hyponormal operators are subscalar, J. Operator Theory 12 (1984), 385-395. MR 85h:47027

8. Y. Chu, Semihyponormal operators are subscalar, Northeastern Math. J. 4 (1988), 145-148. MR 90c:47039

9. Lin Chen, Yan Zikun and Ruan Yingbin, Common properties of operators $R S$ and $S R$ and p-hyponormal operators, Integr. Equat. Oper. Th. 43 (2002), 313-325.

10. B. A. Barnes, Common operator theory of the linear operators $R S$ and $S R$, Proc. Amer. Math. Soc. 126 (1998), 1055-1061. MR 98f:47003

11. Ruan Yingbin and Yan Zikun, Spectral structure and subdecomposability of p-hyponormal operators, Proc. Amer. Math. Soc. 128 (2000), 2069-2074. MR 2000m:47029

12. M. Chō and T. Huruya, $p$-hyponormal operators for $0<p<\frac{1}{2}$, Commentationes Math. 33 (1993), 23-29. MR 95b:47021

Department of Mathematics, Fujian Normal University, Fuzhou, 350007, People's Republic of China

Department of Mathematics, University of Xiamen, Xiamen, 361005, People's RepubLIC OF ChINA

E-mail address: ruanyingbin@263.net

Department of Mathematics, Fujian Normal University, Fuzhou, 350007, People's Republic of China 